\title{
Adaptive Control of a Transport Aircraft Using Differential Thrust
}

\author{
Vahram Stepanyan* \\ Mission Critical Technologies Inc, NASA Ames Research Center, Moffett Field, CA 94035, \\ Kalmanje Krishnakumar ${ }^{\dagger}$ and Nhan Nguyen ${ }^{\ddagger}$ \\ NASA Ames Research Center, Moffett Field, CA 94035
}

\begin{abstract}
The paper presents an adaptive control technique for a damaged large transport aircraft subject to unknown atmospheric disturbances such as wind gust or turbulence. It is assumed that the damage results in vertical tail loss with no rudder authority, which is replaced with a differential thrust input. The proposed technique uses the adaptive prediction based control design in conjunction with the time scale separation principle, based on the singular perturbation theory. The application of later is necessitated by the fact that the engine response to a throttle command is substantially slow that the angular rate dynamics of the aircraft. It is shown that this control technique guarantees the stability of the closed-loop system and the tracking of a given reference model. The simulation example shows the benefits of the approach.
\end{abstract}

\section{Introduction}

The survivability of damaged aircraft is one of the main research topics in the aviation safety research. The catastrophic loss of control of American Airlines Flight 587 illustrates the degree of hazards we can expect due to structural failures of airframe components. ${ }^{2}$ Nevertheless, there are many stories in aviation history of aircraft surviving after suffering major structural damage to their airframes. The recent example of survived aircraft is the DHL A300 cargo flight, which completely lost the hydraulics after it was struck by a missile after departing from Baghdad. ${ }^{1}$ Pilots were able to stabilize and to land the aircraft using differential thrust.

Using differential thrust as an emergency substitute for failed control surfaces has been a research topic after the development of Propulsion-controlled aircraft (PCA) system at NASA Dryden Research Center, which was first evaluated on a piloted B-720 simulation. ${ }^{9}$ The research on the PCA system continued at NASA Dryden and Ames Research Centers in simulations as well as in actual flight test for different flight platforms. ${ }^{4}$ The objective of the PCA system is to provide a necessary thrust command for each engine for emergency flight control in response to pilots flightpath input, assuming that each engine can be controlled individually.

The majority of results in this field are based on known model of the vertical tail loss aircraft, and derive thrust control command using conventional control methods (see for example References ${ }^{3,7,10,21}$ ). In Reference ${ }^{14}$ an adaptive control scheme is used to control the aircraft in the presence of unknown actuator failure, when differential trust is used to generate necessary moment. In all this cases the engine dynamics are ignored, despite the fact that the engine response has a significant lag, especially in law trust levels.

In this paper we consider a propulsion control problem for a transport aircraft with vertical tail loss, taking into account the aircraft's engine dynamics. It is assumed that the damage alters the stability and control derivatives and mass properties of the aircraft. In addition, the aircraft dynamic model involves modeling uncertainties that account for aerodynamic forces and moments, and external disturbances. Since

\footnotetext{
*Senior Scientist, Mission Critical Technologies Inc., Senior Member AIAA, vahram.stepanyan@nasa.gov

${ }^{\dagger}$ Principal Investigator, Integrated Resilient Aircraft Control Project, Intelligent Systems Division, Associate Fellow AIAA, kalmanje.krishnakumar@nasa.gov

${ }^{\ddagger}$ Project Scientist, Integrated Resilient Aircraft Control Project, Intelligent Systems Division, Associate Fellow AIAA, nhan.t.nguyen@nasa.gov
} 
the aircraft is missing the rudder, it is assumed that the necessary yaw moment for the directional control is generated through the differential thrust of the engines, the dynamics of which is know to be much slower than the rotational dynamics of the aircraft. Moreover, it is assumed that the engine dynamics can be modeled as an unknown stable system with throttle input and thrust output, which can be measured (or very accurate estimates are available). Our adaptive control approach uses several ideas from $L_{1}$ adaptive control, ${ }^{5},{ }^{6}$ singular perturbation method ${ }^{13}$ and adaptive time scale separation ${ }^{12}$ to derive aileron, elevator and right and left engine throttle commands to track a given reference command. It is based on the state prediction model based control design, which makes it possible to introduce a low-pass filter in the control channel to suppress the high frequency oscillations that may be generated by adaptive laws. ${ }^{5}$ For the roll and pitch channels this approach generates acceptable control signals. For the yaw channel the necessary differential throttle input has to be generated as an output feedback signal of a cascaded system, comprised of the yaw dynamics and engine dynamics. Therefore, it is tempting to use the the control architecture from the Reference ${ }^{6}$ that allows to introduce a simple model for the engine dynamics, thus simplifying the output feedback adaptive design. However, this approach guarantees good tracking when the model is sufficiently fast, which can not be justified for the slow engine dynamics. Therefore, we propose to use the singular perturbations method in adaptive settings, which is based on the prediction model as well. ${ }^{12}$ The required differential thrust is computed to control the prediction model, and the differential throttle command is computed via the time scale separation, which is proved to be valid for the prediction models constructed for the yaw rate dynamics and engine dynamics. Afterwards, the closeness of the models to corresponding dynamics are proved. Also, it is proved that the closed-loop system is stable and the tracking of the reference commands can be achieved in steady state as well as in the transient.

The rest of the paper is organized as follows. In Section II we give the problem characteristics and assumptions, and formulate the control objective. The engine dynamics is discussed in Section III. The corresponding error dynamics are derived in Section IV. Section V presents the control design steps, and the stability analysis is presented in Section ??. Simulation examples are presented in Section VI, and the paper is concluded by Section VII. Throughout the paper bold symbols are used for vectors and small letters for scalars.

\section{Problem Formulation}

Let the position vectors of the center of gravity $O_{0}$ of undamaged aircraft be $\boldsymbol{r}_{0}$ in some inertial frame $F_{I}$. When the damage occurs, the center of gravity shifts to a new position $O_{1}$, given by the vector $\boldsymbol{r}_{1}$ in the same inertial frame. It is assumed that the shift is constant and is described by the vector $\boldsymbol{\rho}=\boldsymbol{r}_{1}-\boldsymbol{r}_{0}$. As a consequence of this shift a coupling appears between the aircraft's linear and angular accelerations. The corresponding dynamic equations are derived in ${ }^{16}$ and have the following form:

$$
\begin{aligned}
\boldsymbol{F} & =m(\dot{\boldsymbol{v}}+\boldsymbol{\omega} \times \boldsymbol{v})+m \boldsymbol{\omega} \times(\boldsymbol{\omega} \times \boldsymbol{\rho})+m \dot{\boldsymbol{\omega}} \times \boldsymbol{\rho} \\
\boldsymbol{M} & =J_{0} \dot{\boldsymbol{\omega}}+\boldsymbol{\omega} \times J_{0} \boldsymbol{\omega}+m \boldsymbol{\omega} \times(\boldsymbol{\rho} \times \boldsymbol{v})+m \boldsymbol{v} \times(\boldsymbol{\omega} \times \boldsymbol{\rho})+m \boldsymbol{\rho} \times \dot{\boldsymbol{v}}-\boldsymbol{\rho} \times \boldsymbol{W}
\end{aligned}
$$

These equations are written with respect to a body frame $F_{B}$, which is attached to the nominal center of gravity $O_{0}$, with the longitudinal axis through the aircraft's nose. In these equations, $\boldsymbol{v}$ is the velocity vector of the point $O_{0}$ and $\boldsymbol{\omega}$ is the aircraft's angular rate vector, both expressed in the frame $F_{B}, \boldsymbol{F}$ and $\boldsymbol{M}$ represent the sum of external forces and moments in the same frame, and $\boldsymbol{W}$ is the gravity force. $m=m^{*}+\Delta m$ is the unknown mass of the damaged aircraft, where the mass change $\Delta m$ from the known nominal mass $m^{*}$ is negative. $J_{0}$ is the unknown moment of inertia of the damaged aircraft, computed in the frame $F_{B}$. Substituting $\dot{\boldsymbol{v}}$ from the first equation in (1) into the second one, after some algebra the angular rate equation can be written as

$$
\boldsymbol{M}-\boldsymbol{\rho} \times \boldsymbol{F}+\boldsymbol{\rho} \times \boldsymbol{W}=\left(J_{0}+m \boldsymbol{\rho} \boldsymbol{\rho}^{\top}-m \mathbb{I}_{3 \times 3} \boldsymbol{\rho}^{\top} \boldsymbol{\rho}\right) \dot{\boldsymbol{\omega}}+\boldsymbol{\omega} \times J_{0} \boldsymbol{\omega}+m \boldsymbol{\rho}^{\top} \boldsymbol{\omega}(\boldsymbol{\rho} \times \boldsymbol{\omega}) .
$$

We notice that $J=J_{0}+m \boldsymbol{\rho} \boldsymbol{\rho}^{\top}-m \mathbb{I}_{3 \times 3} \boldsymbol{\rho}^{\top} \boldsymbol{\rho}$ is the moment of inertia of the damaged aircraft computed in the parallel to $F_{B}$ frame attached to the new center of gravity $O_{1}$. We decompose the external force $F$ into the sum of the aerodynamic force $\boldsymbol{F}_{A}$, gravity force $\boldsymbol{W}$ and the collective thrust $\boldsymbol{T}$ generated by left and right engines.

$$
\boldsymbol{F}=\boldsymbol{F}_{A}+\boldsymbol{W}+\boldsymbol{T}
$$


Similarly, we decompose the external moment $\boldsymbol{M}$ into the sum of the aerodynamic moment $\boldsymbol{M}_{A}$, the moment of the gravity force $\boldsymbol{W}$ and the moment generated by the differential thrust $\Delta \boldsymbol{T}$. The aerodynamic moment $\boldsymbol{M}_{A}$ is further decomposed into the sum of the moment generated by the control surfaces $\boldsymbol{M}_{\delta}$ and the moment generated by the rest of the aircraft $\boldsymbol{M}_{b}$.

$$
M_{A}=M_{b}+M_{\delta}+\rho \times \boldsymbol{W}+\boldsymbol{a} \times \Delta \boldsymbol{T} .
$$

where $\boldsymbol{a}$ denotes the radius vector of the center of the right engine thrust vector. Substituting the force and moment equations into (2), the angular rate dynamics can be expressed in the following form

$$
J \dot{\boldsymbol{\omega}}=-\boldsymbol{\omega} \times J_{0} \boldsymbol{\omega}+m \boldsymbol{\rho}^{\top} \boldsymbol{\omega}(\boldsymbol{\rho} \times \boldsymbol{\omega})-\boldsymbol{\rho} \times(\boldsymbol{T}-\boldsymbol{W})+\boldsymbol{M}_{\delta}+\boldsymbol{a} \times \Delta \boldsymbol{T}+\boldsymbol{M}_{b}-\boldsymbol{\rho} \times \boldsymbol{F}_{A},
$$

Next, we substitute $\dot{\boldsymbol{\omega}}$ into first equation in (1) and obtain a force equation in the following form

$$
\begin{aligned}
\dot{\boldsymbol{v}} & =\frac{\boldsymbol{F}}{m}-\boldsymbol{\omega} \times \boldsymbol{v}-\boldsymbol{\omega} \boldsymbol{\omega}^{\top} \boldsymbol{\rho}-J^{-1}\left(-\boldsymbol{\omega} \times J_{0} \boldsymbol{\omega}+m \boldsymbol{\rho}^{\top} \boldsymbol{\omega}(\boldsymbol{\rho} \times \boldsymbol{\omega})\right. \\
& \left.-\boldsymbol{\rho} \times(\boldsymbol{T}-\boldsymbol{W})+\boldsymbol{M}_{\delta}+\boldsymbol{a} \times \Delta \boldsymbol{T}+\boldsymbol{M}_{b}-\boldsymbol{\rho} \times \boldsymbol{F}_{A}\right) \times \boldsymbol{\rho}
\end{aligned}
$$

The complete dynamics of the damaged aircraft includes also the orientation equations and navigation equations. However, in this paper we are not interested in navigation problems as well as the yaw orientation equation. That is, the damaged aircraft's dynamics are given by the equations (5), (6) and

$$
\begin{aligned}
\dot{\phi} & =p+q \sin \phi \tan \theta+r \cos \phi \tan \theta \\
\dot{\theta} & =q \cos \phi-r \sin \phi
\end{aligned}
$$

We assume that the thrust vector is aligned with the longitudinal axis, that is $\boldsymbol{T}=\left[\begin{array}{lll}T & 0 & 0\end{array}\right]^{\top}$ and $\Delta \boldsymbol{T}=\left[\begin{array}{lll}\Delta T & 0 & 0\end{array}\right]^{\top}$, where $T$ and $\Delta T$ are the magnitudes of the collective thrust and differential thrust respectively. This quantities are subject to the following constraints

$$
\begin{aligned}
0 & \leq \quad T \\
-\frac{1}{2} T & \leq T_{\max } \\
& \leq \frac{1}{2} T,
\end{aligned}
$$

where $T_{\max }$ is the given maximum collective thrust that can be generated by engines. The second inequality in (8) implies that the one sided thrust is allowed during the maneuvers.

It is assumed that the damage of the aircraft results in the vertical tail loss. This in turn results in the directional instability of the aircraft with no rudder authority. The objective is to control the aircraft using the available control surfaces and the differential thrust.

\section{Engine Dynamics}

In general, typical aircraft engine dynamics are highly nonlinear and of distributed character. The response depends not only on the throttle input and other combustion parameters, but also on the thrust level. Figure 1 displays some examples of engine response data to a full throttle input at different altitudes with initial thrust levels corresponding to trim conditions for a generic transport aircraft model. ${ }^{8}$

It also shows that for the low thrust level the initial delay in response is much larger than on the high thrust level, and the response is faster at law altitudes. The forms of the engine response data suggest that it is possible to approximate the engine dynamics by a stable linear system with time delay, which afterwards can be transformed into a high order linear time invariant system using Pade approximations. Figure 2 shows the forth order linear approximation of the engine response at low trust level, which corresponds to a high altitude cruise. It can be seen that the high order linear time invariant approximation describes the engine response with sufficient accuracy. Therefore, for the purpose of this paper it is assumed that the thrust generated by engines can be described as the output of a slow but stable minimum phase linear system with known relative degree and with a single throttle input.

Since the left and right engines are assumed to be identical, the collective thrust and the differential thrust are described by the same dynamic model

$$
\begin{aligned}
\epsilon^{-1} \dot{\boldsymbol{x}}_{e}(t) & =A_{e} \boldsymbol{x}_{e}(t)+\boldsymbol{b}_{e}\left(u_{e}(t)+d_{e}(t)\right) \\
y_{e}(t) & =\boldsymbol{c}_{e} \boldsymbol{x}_{e}(t)
\end{aligned}
$$




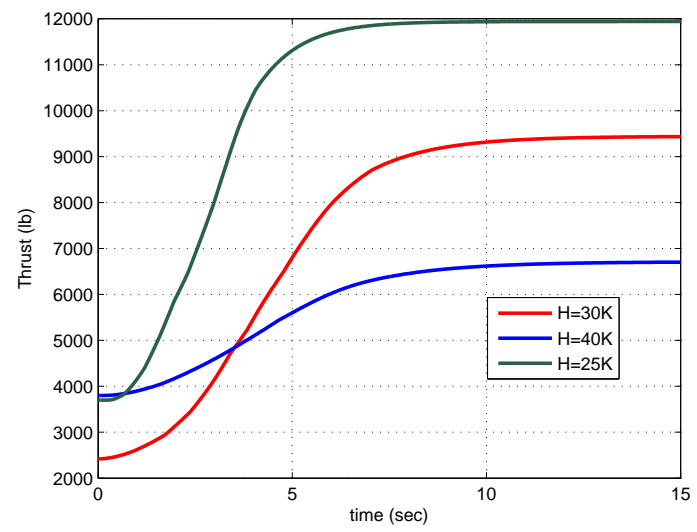

Figure 1. Engine response data for different altitudes and initial thrust level

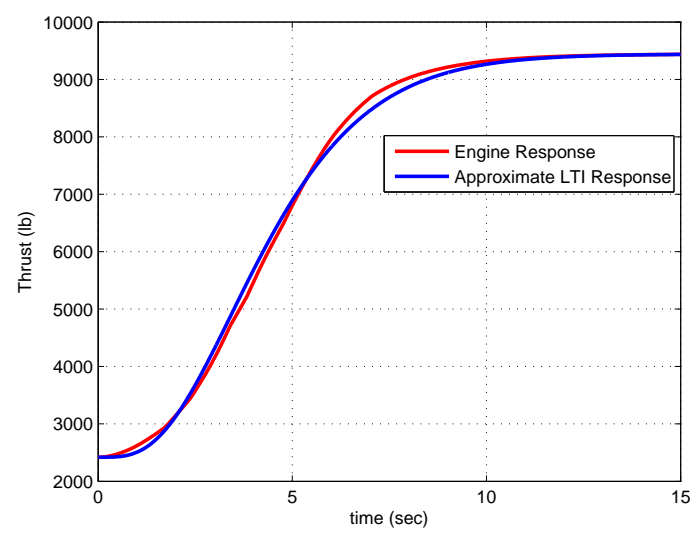

Figure 2. Engine response data and 4th order linear approximation response at altitude $30000 \mathrm{ft}$

where $\boldsymbol{x}_{e}(t)$ is the model's state, $A_{e}, B_{e}, C_{e}$ are matrices of compatible dimensions with $A_{e}$ being Hurwitz, $\epsilon$ is a small parameter indicating that the engines dynamics are slow in the real time scale, and $d_{e}(t)$ is a bounded external disturbance. For the collective thrust generation $y_{e}(t)=T(t), u_{e}(t)=\delta_{T}(t)$, where $\delta_{T}(t)$ is the collective throttle input, and for the differential thrust generation $y_{e}(t)=\Delta T(t), u_{e}(t)=\delta_{\Delta T}(t)$, where $\delta_{\Delta T}(t)$ is the differential throttle input. The throttle inputs to the left and right engines are defined as

$$
\begin{aligned}
\delta_{T_{L}} & =\frac{1}{2}\left[\delta_{T}+\delta_{\Delta T}\right] \\
\delta_{T_{R}} & =\frac{1}{2}\left[\delta_{T}-\delta_{\Delta T}\right]
\end{aligned}
$$

\section{Error Dynamics}

With the vertical tail loss the aircraft is still symmetrical with respect to the $x z$ plane, that is the center of gravity shift has zero $y$ coordinate and $J_{y z}=0$. Therefore, it is possible to decouple the longitudinal and the lateral-directional dynamics. However, coupling remains in the control inputs via the thrust generation. The point will be clarified shortly. Keeping this in mind, we linearize the aircraft's dynamics about a trim point that corresponds to a damaged state, and write the longitudinal and lateral-directional dynamics separately

$$
\dot{\boldsymbol{x}}_{L}(t)=A_{L} \boldsymbol{x}_{L}(t)+B_{L}\left[\Lambda_{L} \boldsymbol{u}_{L}(t)+\boldsymbol{f}_{L}\left(\boldsymbol{x}_{L}(t), \boldsymbol{x}_{D}(t)\right)+\boldsymbol{d}_{L}(t)\right],
$$


where the longitudinal state $\boldsymbol{x}_{L}$ and the control input $\boldsymbol{u}_{L}$ are defined as

$$
\boldsymbol{x}_{L}=\left[\begin{array}{llll}
\Delta V & \Delta \alpha & q & \Delta \theta
\end{array}\right]^{\top}, \boldsymbol{u}_{L}=\left[\begin{array}{ll}
T & \delta_{e}
\end{array}\right]^{\top},
$$

the control allocation matrix $A_{L} \in \mathbb{R}^{4 \times 2}$ is assumed two ne known, matrices matrix $A_{L} \in \mathbb{R}^{4 \times 4}$ and $\Lambda_{L} \in$ $\mathbb{R}^{2 \times 2}$ are unknown, where $\Lambda_{L}$ is diagonal positive definite, and represents the unknown control effectiveness. the nominal value of $\Lambda_{L}$ is the identity matrix. The continuous and locally Lipschitz function $\boldsymbol{f}_{L}\left(\boldsymbol{x}_{L}, \boldsymbol{x}_{D}\right)$ represents modeling uncertainties and the bounded time function $\boldsymbol{d}_{L}(t)$ is added to account for external disturbances such as the turbulence or wind gust. In the above equation,

$$
\boldsymbol{x}_{L}=\left[\begin{array}{llll}
\beta & r & p & \phi
\end{array}\right]^{\top}
$$

is the lateral-directional state that satisfies the equation

$$
\dot{\boldsymbol{x}}_{D}(t)=A_{D} \boldsymbol{x}_{D}(t)+B_{D}\left[\Lambda_{D} \boldsymbol{u}_{D}(t)+\boldsymbol{f}_{D}\left(\boldsymbol{x}_{L}(t), \boldsymbol{x}_{D}(t)\right)+\boldsymbol{d}_{D}(t)\right],
$$

where $\boldsymbol{u}_{D}=\left[\begin{array}{ll}\delta_{a} & \Delta T\end{array}\right]^{\top}$ is the lateral-directional control input, the matrices $A_{D}, B_{D}, \Lambda_{D}$ and functions $\boldsymbol{f}_{D}\left(\boldsymbol{x}_{L}, \boldsymbol{x}_{D}\right), \boldsymbol{d}_{D}(t)$ have the same meaning as in the case of longitudinal dynamics.

Remark 1 . In the above equations the collective thrust $T$ and the differential thrust $\Delta T$ are considered as independent input signals that are generated through the engines, the required thrusts of which are defined according to the equations

$$
\begin{gathered}
T_{\text {left }}=T+\frac{1}{2} \Delta T \\
T_{\text {right }}=T-\frac{1}{2} \Delta T,
\end{gathered}
$$

where $T$ and $\Delta T$ are considered commands generated from the perspective of controlling the longitudinal and lateral-directional dynamics respectively. In this case the collective thrust remains intact while the engines undergo changes to generate the required differential thrust. Therefore, the decoupling of the dynamics is complete. However, as we will see in the simulations, this way of generating the differential thrust is not always the best solution, since for the vertical tail loss aircraft the directional stability is a priority and all possible control capabilities must be used for this purposes. The alternative is to control the engines separately to achieve maximal differential thrust at each operating point in the expense of altering the desired collective thrust, which cannot be considered as an independent control signal any more. The excess collective thrust will affect the longitudinal motion, which can be compensated for after the directional stability is archived.

We construct the reference models for the longitudinal and lateral-directional dynamics that meet the desired performance characteristics as follows. Let $A_{L}=A_{L}^{0}+\Delta A_{L}$ and $A_{D}=A_{D}^{0}+\Delta A_{D}$, where $A_{L}^{0}$ and $A_{D}^{0}$ are known matrices such that the pairs $\left(A_{L}, B_{L}\right)$ and $\left(A_{L}, B_{L}\right)$ are controllable, and $\Delta A_{L}$ and $\Delta A_{D}$ are the uncertainties that satisfy the following model matching assumption.

Assumption 1 . For Hurwitz matrices $A_{L m}$ and $A_{D m}$ there exist constant matrices $K_{L x}^{*} \in \mathbb{R}^{2 \times 4}, K_{L r}^{*} \in$ $\mathbb{R}^{2 \times 2}, K_{D x}^{*} \in \mathbb{R}^{2 \times 4}$ and $K_{D r}^{*} \in \mathbb{R}^{2 \times 2}$ such that

$$
\begin{aligned}
B_{L} K_{L x}^{*}=\Delta A_{L} & B_{D} K_{D x}^{*}=\Delta A_{D} \\
B_{L} K_{L r}^{*}=B_{L m} & B_{D} K_{D r}^{*}=B_{D m} .
\end{aligned}
$$

Remark 2. The true knowledge of the matrices $K_{L x}^{*}, K_{L r}^{*}, K_{D x}^{*}$ and $K_{D r}^{*}$ is not required, only their existence is assumed.

The reference models are obtained from the corresponding dynamics in the absence of uncertainties and disturbances by means of the linear controls

$$
\begin{aligned}
\boldsymbol{u}_{L}^{0}(t) & =-K_{L x}^{0} \boldsymbol{x}_{L}(t)+K_{L r}^{0} \boldsymbol{r}_{L}(t) \\
\boldsymbol{u}_{D}^{0}(t) & =-K_{D x}^{0} \boldsymbol{x}_{D}(t)+K_{D r}^{0} \boldsymbol{r}_{D}(t),
\end{aligned}
$$


where the gains $K_{L x}^{0}$, and $K_{D x}^{0}$ are chosen such the the matrices $A_{L m}=A_{L}^{0}-B_{L} K_{L x}^{0}$ and $A_{D m}=A_{D}^{0}$ $B_{D} K_{D x}^{0}$ are Hurwitz. The reference models for the longitudinal dynamics and lateral directional dynamic have form

$$
\begin{aligned}
& \dot{\boldsymbol{x}}_{L m}(t)=A_{L m} \boldsymbol{x}_{L m}(t)+B_{L m} \boldsymbol{r}_{L}(t) \\
& \dot{\boldsymbol{x}}_{D m}(t)=A_{D m} \boldsymbol{x}_{D m}(t)+B_{D m} \boldsymbol{r}_{D}(t),
\end{aligned}
$$

where the $\boldsymbol{r}_{L}(t)$ and $\boldsymbol{r}_{D}(t)$ are external command signals.

We take the adaptive augmentation approach in the control law design. That is the control signals are represented as

$$
\begin{aligned}
\boldsymbol{u}_{L}(t) & =\boldsymbol{u}_{L}^{0}(t)+\boldsymbol{u}_{L}^{a d}(t) \\
\boldsymbol{u}_{D}(t) & =\boldsymbol{u}_{D}^{0}(t)+\boldsymbol{u}_{D}^{a d}(t),
\end{aligned}
$$

where $\boldsymbol{u}_{L}^{0}(t)$ and $\boldsymbol{u}_{D}^{0}(t)$ baseline control signals in (16), and $\boldsymbol{u}_{L}^{a d}(t), \boldsymbol{u}_{D}^{a d}(t)$ are adaptive augmentations to be designed later. Denoting the longitudinal tracking error by $\boldsymbol{e}_{L}(t)=\dot{\boldsymbol{x}}_{L}(t)-\dot{\boldsymbol{x}}_{L m}(t)$, the error dynamics can be written in the

$$
\dot{\boldsymbol{e}}_{L}(t)=A_{L m} \boldsymbol{e}_{L}(t)+B_{L}\left[\Lambda_{L}\left(\boldsymbol{u}_{L}(t)-\boldsymbol{u}_{L}^{0}(t)\right)+K_{L x} \boldsymbol{x}_{L}(t)+K_{L r} \boldsymbol{r}_{L}(t)+\boldsymbol{f}_{L}\left(\boldsymbol{x}_{L}(t), \boldsymbol{x}_{D}(t)\right)+\boldsymbol{d}_{L}(t)\right],
$$

where $K_{L x}=\left(\mathbb{I}-\Lambda_{L}\right) K_{L x}^{0}+K_{L x}^{*}$ and $K_{L r}=\left(\mathbb{I}-\Lambda_{L}\right) K_{L r}^{0}+K_{L r}^{*}$. Similarly, the lateral-directional tracking error dynamics can be written as

$\dot{\boldsymbol{e}}_{D}(t)=A_{D m} \boldsymbol{e}_{D}(t)+B_{D}\left[\Lambda_{D}\left(\boldsymbol{u}_{D}(t)-\boldsymbol{u}_{D}^{0}(t)\right)+K_{D x} \boldsymbol{x}_{D}(t)+K_{D r} \boldsymbol{r}_{D}(t)+\boldsymbol{f}_{D}\left(\boldsymbol{x}_{L}(t), \boldsymbol{x}_{D}(t)\right)+\boldsymbol{d}_{D}(t)\right],(20$

where $\boldsymbol{e}_{D}(t)=\dot{\boldsymbol{x}}_{D}(t)-\dot{\boldsymbol{x}}_{D m}(t), K_{D x}=\left(\mathbb{I}-\Lambda_{D}\right) K_{D x}^{0}+K_{D x}^{*}$ and $K_{D r}=\left(\mathbb{I}-\Lambda_{D}\right) K_{D r}^{0}+K_{D r}^{*}$.

Remark 3 . We notice that the error dynamics (19) and (20) are in the form considered earlier in the literature and the suitable adaptive control design techniques guaranteeing tracking both in the steady state and in transient can be found in References. ${ }^{20,22}$ Next step would be to design suitable laws for the throttle inputs in order to generate the required collective and differential thrust signals designed in the previous step. Whereas for the longitudinal design the direct application of any design approach would perform satisfactory, for the lateral-directional design it leads to a throttle command that involves high gain terms. ${ }^{19}$ The reason is that the engine dynamics are much faster than the fungoid mode and much slower than the rotational dynamics controlled by the rudder deflection. One of the remedies for the situation is the principle of the time scale separation, which is based on the Tikhonov's theorem. ${ }^{13}$ This theorem requires some exponential stability properties for the cascaded systems under the consideration. However, no exponential stability of the error system (20) can be establish without the parameter convergence, which cannot be guaranteed in general, especially when the unknown nonlinearities are involved. This prevents the direct application of for the time scale separation. As an intermediate step we follow the approach from Reference ${ }^{12}$ and design suitable prediction models for which the time scale separation theorem can be applied. Since the longitudinal dynamics do not require two time scale separation, we drop them from further consideration, assuming that the suitable longitudinal design is already available, and the longitudinal variables reside in the suitable bounded regions. That is to design control laws for the lateral-directional dynamics all longitudinal variables are assumed to be bounded.

Since $\boldsymbol{f}_{D}\left(\boldsymbol{x}_{L}, \boldsymbol{x}_{D}\right)$ is continuous, we approximate it on a compact set $\Omega_{\boldsymbol{x}_{L}} \times \Omega_{\boldsymbol{x}_{D}}$ by any known approximation method. Here, we use normalized radial bases functions ${ }^{11}$ to approximate $\boldsymbol{f}(\boldsymbol{v}, \boldsymbol{\omega})$ by a linear in parameter neural network on the compact set $\Omega_{x_{L}} \times \Omega_{x_{D}}$. That is we write

$$
\boldsymbol{f}_{D}\left(\boldsymbol{x}_{L}, \boldsymbol{x}_{D}\right)=W^{\top} \boldsymbol{\varphi}\left(\boldsymbol{x}_{L}, \boldsymbol{x}_{D}\right)+\varepsilon\left(\boldsymbol{x}_{L}, \boldsymbol{x}_{D}\right),
$$

where $W \in \mathbb{R}^{N \times 2}$ is the unknown optimal weight matrix, which minimize the norm difference $\| \boldsymbol{f}_{D}\left(\boldsymbol{x}_{L}, \boldsymbol{x}_{D}\right)$ $W^{\top} \boldsymbol{\varphi}\left(\boldsymbol{x}_{L}, \boldsymbol{x}_{D}\right) \|, \boldsymbol{\varphi}\left(\boldsymbol{x}_{L}, \boldsymbol{x}_{D}\right)$ is the vector of basis functions and $\varepsilon\left(\boldsymbol{x}_{L}, \boldsymbol{x}_{D}\right)$ is function reconstruction error that is uniformly bounded by a constant $\varepsilon^{*}$. We notice that from the above considerations the relationship $\boldsymbol{x}_{L} \in$ $\Omega_{\boldsymbol{x}_{L}}$ is satisfied, and $\boldsymbol{x}_{D} \in \Omega_{\boldsymbol{x}_{D}}$ will be guaranteed by the control design. Substituting the approximation (21) into the error dynamics (20) we obtain

$$
\dot{\boldsymbol{e}}(t)=A_{m} \boldsymbol{e}(t)+B\left[\Lambda \boldsymbol{u}(t)+K_{x} \boldsymbol{x}(t)+K_{r} \boldsymbol{r}(t)+W^{\top} \boldsymbol{\varphi}(t)+\boldsymbol{\sigma}(t)\right],
$$


where for the notational simplicity we drop the subscript " $D$ " and introduce notations $\boldsymbol{u}(t)=\boldsymbol{u}_{D}(t)-\boldsymbol{u}_{D}^{0}(t)$, $\boldsymbol{\varphi}(t)=\boldsymbol{\varphi}\left(\boldsymbol{x}_{L}(t), \boldsymbol{x}_{D}(t)\right), \boldsymbol{\sigma}(t)=\boldsymbol{\varepsilon}\left(\boldsymbol{x}_{L}(t), \boldsymbol{x}_{D}(t)\right)+\boldsymbol{d}_{D}(t)$, where $\boldsymbol{\sigma}(t)$ is bounded componentwise by some positive constant vector $\boldsymbol{\sigma}^{*}$ as long as the relationship $\left(\boldsymbol{x}_{L}(t), \boldsymbol{x}_{D}(t)\right) \in \Omega_{\boldsymbol{x}_{L}} \times \Omega_{\boldsymbol{x}_{D}}$ holds. Also, since $A_{m}$ is a Hurwitz matrix there exists a symmetric positive definite matrix $P$ that satisfies the Lyapunov equation

$$
A_{m}^{\top} P+P A_{m}=-Q
$$

for some symmetric positive definite matrix $Q$.

\section{Control Design}

In this section we construct the stabilizing control law for the cascaded system that is comprised od the error dynamics (22) and the engine dynamics (9). To this end we assume that the engine dynamics is of full relative degree $n$, and therefore can be represented in the normal form without internal dynamic

$$
\begin{aligned}
\varepsilon^{-1} \dot{\boldsymbol{x}}_{e}(t) & =\left(A_{0}-\boldsymbol{b}_{0} \boldsymbol{k}^{\top}\right) \boldsymbol{x}_{e}(t)+\boldsymbol{b}_{0}\left[\omega u_{e}(t)+d_{e}(t)\right] \\
y_{e}(t) & =\boldsymbol{c}_{0} \top \boldsymbol{x}_{e}(t) .
\end{aligned}
$$

where the triplet $\left(A_{0}, \boldsymbol{b}_{0}, \boldsymbol{c}_{0}\right)$ is the canonical form representation of chain of $n$ integrators, $\boldsymbol{k}$ is an unknown constant vector, $\omega$ is the unknown constant control effectiveness of known sign (assume is positive), and $y_{e}(t)=\Delta T(t)$ and $u_{e}(t)=\Delta \delta_{T}(t)$.

\section{A. Prediction Models}

The prediction model for the lateral-directional tracking error dynamics is introduced according to equation

$$
\dot{\hat{\boldsymbol{e}}}(t)=A_{m} \hat{\boldsymbol{e}}(t)+B\left[\hat{\Lambda}(t) \overline{\boldsymbol{u}}(t)+\hat{K}_{x}^{\top}(t) \boldsymbol{x}(t)+\hat{K}_{r}^{\top}(t) \boldsymbol{r}(t)+\hat{W}^{\top}(t) \boldsymbol{\varphi}(t)+\boldsymbol{h}(t)\right],
$$

where the variables with "hat" are the estimates of the corresponding unknown constant parameters, $\overline{\boldsymbol{u}}(t)=\left[\delta_{a}(t)-\delta_{a}^{0}(t) \hat{\Delta T}(t)-\Delta T^{0}(t)\right]^{\top}, \delta_{a}^{0}(t)$ and $\Delta T^{0}(t)$ are baseline aileron deflection and differential thrust commands, $\hat{\Delta} T(t)$ is the prediction of the differential thrust command to be design shortly, $\boldsymbol{h}(t)=\left[\begin{array}{ll}\hat{\sigma}_{1}(t) \operatorname{sign}\left(\chi_{1}(t)\right) & \hat{\sigma}_{2}(t) \operatorname{sign}\left(\chi_{2}(t)\right)\end{array}\right]^{\top}, \hat{\boldsymbol{\sigma}}(t) \triangleq\left[\hat{\sigma}_{1}(t) \hat{\sigma}_{2}(t)\right]^{\top}$ is the estimate of the unknown bound $\boldsymbol{\sigma}^{*}$ and $\boldsymbol{\chi}(t) \triangleq\left[\chi_{1}(t) \chi_{2}(t)\right]^{\top}=B^{\top} P \tilde{\boldsymbol{e}}(t)$, and $\tilde{\boldsymbol{e}}(t)=\boldsymbol{e}(t)-\hat{\boldsymbol{e}}(t)$ is the prediction error. The estimates $\hat{\Lambda}(t)$, $\hat{K}_{x}(t), \hat{K}_{r}(t), \hat{W}^{\top}(t)$ and $\hat{\boldsymbol{\sigma}}(t)$ are given by the adaptive laws

$$
\begin{aligned}
\dot{\hat{\Lambda}}(t) & =\gamma_{1} \Pi\left\{\hat{\Lambda}(t), \boldsymbol{u}(t) \boldsymbol{\chi}^{\top}(t)\right\} \\
\dot{\hat{K}}_{x}(t) & =\gamma_{2} \boldsymbol{x}(t) \boldsymbol{\chi}^{\top}(t) \\
\dot{\hat{K}}_{r}(t) & =\gamma_{3} \boldsymbol{r}(t) \boldsymbol{\chi}^{\top}(t) \\
\dot{\hat{W}}_{(t)} & =\gamma_{4} \boldsymbol{\varphi}(t) \boldsymbol{\chi}^{\top}(t) \\
\dot{\hat{\sigma}}_{r}(t) & =\gamma_{5}|\chi(t)|
\end{aligned}
$$

where $\gamma_{1}>0, \gamma_{2}>0, \gamma_{3}>0, \gamma_{4}>0$ and $\gamma_{5}>0$ are the adaptation rates, the projection operator ${ }^{17}$ $\Pi(\cdot, \cdot)$ is introduced to keep the estimate $\hat{\Lambda}(t)$ positive, and the absolute value of the vector $\chi(t)$ is defined componentwise. The prediction error dynamics can be written as

$$
\dot{\tilde{\boldsymbol{e}}}(t)=A_{m} \tilde{\boldsymbol{e}}(t)+B\left[\tilde{\Lambda}(t) \boldsymbol{u}(t)+\hat{\Lambda}(t) \tilde{\boldsymbol{u}}(t)+\tilde{K}_{x}^{\top}(t) \boldsymbol{x}(t)+\tilde{K}_{r}^{\top}(t) \boldsymbol{r}(t)+\tilde{W}^{\top}(t) \boldsymbol{\varphi}(t)+\sigma(t)-\boldsymbol{h}(t)\right],
$$

where $\tilde{\Lambda}(t)=\Lambda-\hat{\Lambda}(t), \tilde{K}_{x}(t)=K_{x}-\hat{K}_{x}(t), \tilde{K}_{r}(t)=K_{r}-\hat{K}_{r}(t)$, and $\tilde{W}(t)=W-\hat{W}(t)$ are the parameter estimation errors, $\tilde{\boldsymbol{u}}(t)=[0 \tilde{\Delta T}(t)]^{\top}$ and $\tilde{\Delta T}(t)=\Delta T(t)-\hat{\Delta T}(t)$ is the differential thrust prediction error.

To be able to introduce a suitable prediction model for the engine dynamics in (24), we introduce filters following the conventional linear system parametrization scheme (see for example ${ }^{15,18}$ )

$$
\begin{array}{lll}
\varepsilon^{-1} \dot{\boldsymbol{\xi}}_{1}(t)=\left(A_{0}-\boldsymbol{b}^{0} \boldsymbol{k}_{0}^{\top}\right) \boldsymbol{\xi}_{1}(t)+\boldsymbol{b}^{0} y_{a}(t), & \boldsymbol{\xi}_{1}(0)=0 \\
\varepsilon^{-1} \dot{\boldsymbol{\xi}}_{2}(t)=\left(A_{0}-\boldsymbol{b}^{0} \boldsymbol{k}_{0}^{\top}\right) \boldsymbol{\xi}_{2}(t)+\boldsymbol{b}^{0} u_{e}(t), & \boldsymbol{\xi}_{2}(0)=0 \\
\varepsilon^{-1} \dot{\boldsymbol{\xi}}_{3}(t)=\left(A_{0}-\boldsymbol{b}^{0} \boldsymbol{k}_{0}^{\top}\right) \boldsymbol{\xi}_{3}(t)+\boldsymbol{b}^{0} d_{e}(t), & \boldsymbol{\xi}_{3}(0)=0
\end{array}
$$


where $\boldsymbol{b}^{0}=\left[\begin{array}{llll}1 & b_{2}^{0} & \ldots & b_{n}^{0}\end{array}\right]^{\top}$ is chosen such that the pair $\left(A_{0}, \boldsymbol{b}^{0}\right)$ is controllable and the polynomial $s^{n-1}+$ $b_{2}^{0} s^{n-2}+\cdots+b_{n}^{0}$ is Hurwitz, $\boldsymbol{k}_{0} \in \mathbb{R}^{n}$ is chosen such that $A_{0}-\boldsymbol{b}^{0} \boldsymbol{k}_{0}^{\top}$ is Hurwitz. Then, the differential thrust can be represented as

$$
y_{e}(t)=\boldsymbol{q}^{\top} \boldsymbol{\xi}_{1}(t)+\omega \xi_{21}(t)+\sigma_{e}(t),
$$

where we denote $\boldsymbol{q}=\boldsymbol{k}_{0}-\boldsymbol{k}$ and $\sigma_{e}(t)=\xi_{31}(t)$. Since the filters are stable, from the boundedness of the inputs the boundedness of the outputs follow. That is $\sigma_{f}(t)$ is bounded by some positive constant $\sigma_{f}^{*}$ since $d_{e}(t)$ is assumed to be bounded. The prediction model for the differential thrust is introduced as

$$
\hat{y}_{e}(t)=\hat{\boldsymbol{q}}^{\top}(t) \boldsymbol{\xi}_{1}(t)+\hat{\omega}(t) \xi_{21}(t)+\hat{\sigma}_{e}(t) \operatorname{sign}\left(\chi_{2}(t)\right),
$$

where $\hat{\boldsymbol{q}}(t)$ and $\hat{\omega}(t)$ are the estimates of unknown parameters $\boldsymbol{q}$ and $\omega$ respectively, and $\hat{\sigma}_{f}(t)$ is the estimate of the unknown bound $\sigma_{f}^{*}$. The prediction error $\tilde{y}_{e}(t)=y_{e}(t)-\hat{y}_{e}(t)$ is given by the equation

$$
\tilde{y}_{e}(t)=\tilde{\boldsymbol{q}}^{\top}(t) \boldsymbol{\xi}_{1}(t)+\tilde{\omega}(t) \xi_{21}(t)+\sigma_{e}(t)-\hat{\sigma}_{e}(t) \operatorname{sign}\left(\chi_{2}(t)\right),
$$

where $\tilde{\boldsymbol{q}}(t)=\boldsymbol{q}-\hat{\boldsymbol{q}}(t)$, and $\tilde{\omega}(t)=\omega-\hat{\omega}(t)$ are the parameter estimation errors. The adaptive laws for the estimates $\hat{\boldsymbol{q}}(t)$ and $\hat{\omega}(t)$ in the differential thrust prediction model are defined as

$$
\begin{aligned}
\dot{\hat{\boldsymbol{q}}}(t) & =\gamma_{6}\left[\tilde{y}_{e}(t) \boldsymbol{\xi}_{1}(t)+\hat{\lambda}_{2}(t) \chi_{2}(t) \boldsymbol{\xi}_{1}(t)\right] \\
\dot{\hat{\omega}}(t) & =\gamma_{7} \Pi\left\{\hat{\omega}(t), \tilde{y}_{e}(t) \xi_{21}(t)+\hat{\lambda}_{2}(t) \chi_{2}(t) \xi_{21}(t)\right\} \\
\dot{\hat{\sigma}}_{e}(t) & =\gamma_{8}\left[\left|\tilde{y}_{e}(t)\right|+\hat{\lambda}_{2}(t)\left|\chi_{2}(t)\right|\right],
\end{aligned}
$$

where $\gamma_{6}>0, \gamma_{7}>0$ and $\gamma_{8}>0$ are the adaptation rates, and the projection operator ${ }^{17} \Pi(\cdot, \cdot)$ is introduced to keep $\hat{\omega}(t)$ positive.

The following lemma guarantees the closeness of the prediction models to the corresponding dynamics.

Lemma 1 . The adaptive laws (26) and (32) guarantee boundedness of the estimates $\hat{\Lambda}(t), \hat{K}_{x}(t), \hat{K}_{r}(t)$, $\hat{W}^{\top}(t)$ and $\hat{\boldsymbol{\sigma}}(t)$ as well as $\hat{\boldsymbol{q}}(t), \hat{\omega}(t), \hat{\sigma}_{f}(t)$ and the prediction errors $\tilde{\boldsymbol{e}}(t)$ and $\tilde{y}_{e}(t)$. Moreover, $\tilde{\boldsymbol{e}}(t) \in \mathcal{L}_{2}$ and $\tilde{y}_{e}(t) \in \mathcal{L}_{2}$.

Proof. Consider the following candidate Lyapunov function

$$
\begin{aligned}
V(t) & =\tilde{\boldsymbol{e}}^{\top}(t) P \tilde{\boldsymbol{e}}_{r}(t)+\operatorname{tr}\left\{\gamma_{1}^{-1} \tilde{\Lambda}^{\top}(t) \tilde{\Lambda}(t)+\gamma_{2}^{-1} \tilde{K}_{x}^{\top}(t) \tilde{K}_{x}(t)+\gamma_{3}^{-1} \tilde{K}_{r}^{\top}(t) \tilde{K}_{r}(t)+\gamma_{4}^{-1} \tilde{w}^{\top}(t) \tilde{W}(t)\right\} \\
& +\gamma_{5}^{-1}\left(\boldsymbol{\sigma}_{r}^{*}-\hat{\boldsymbol{\sigma}}_{r}(t)\right)^{\top}\left(\boldsymbol{\sigma}_{r}^{*}-\hat{\boldsymbol{\sigma}}_{r}(t)\right)+\gamma_{6}^{-1} \tilde{\boldsymbol{q}} \top(t) \tilde{\boldsymbol{q}}(t)+\gamma_{7}^{-1} \tilde{\omega}(t) \tilde{\omega}(t)+\gamma_{8}^{-1}\left(\sigma_{e}^{*}-\hat{\sigma}_{e}(t)\right)^{2} .
\end{aligned}
$$

The derivative of $V(t)$ is computed along the trajectories of systems (27), and (26), (31) and (32).

$$
\begin{aligned}
& \dot{V}(t)=-\tilde{\boldsymbol{e}}_{r}^{\top}(t) Q_{r} \tilde{\boldsymbol{e}}_{r}(t)+2 \boldsymbol{\chi}^{\top}(t)\left[\tilde{\Lambda}(t) \boldsymbol{u}(t)+\hat{\Lambda}(t) \tilde{\boldsymbol{u}}(t)+\tilde{K}_{x}^{\top}(t) \boldsymbol{x}(t)+\tilde{K}_{r}^{\top}(t) \boldsymbol{r}(t)+\tilde{W}^{\top}(t) \boldsymbol{\varphi}(t)+\sigma(t)\right. \\
& \text { - } \boldsymbol{h}(t)]+2 \operatorname{tr}\left\{\gamma_{1}^{-1} \tilde{\Lambda}^{\top}(t) \dot{\tilde{\Lambda}}(t)+\gamma_{2}^{-1} \tilde{K}_{x}^{\top}(t) \dot{\tilde{K}}_{x}(t)+\gamma_{3}^{-1} \tilde{K}_{r}^{\top}(t) \dot{\tilde{K}}_{r}(t)+\gamma_{4}^{-1} \tilde{w}^{\top}(t) \dot{\tilde{W}}(t)\right\} \\
& -2 \gamma_{5}^{-1}\left(\boldsymbol{\sigma}^{*}-\hat{\boldsymbol{\sigma}}(t)\right)^{\top} \dot{\hat{\boldsymbol{\sigma}}}(t)-2 \gamma_{6}^{-1} \tilde{\boldsymbol{q}}^{\top}(t) \dot{\hat{\boldsymbol{q}}}(t)-2 \gamma_{7}^{-1} \tilde{\omega}(t) \dot{\hat{\omega}}(t)-2 \gamma_{8}^{-1}\left(\sigma_{e}^{*}-\hat{\sigma}_{e}(t)\right) \dot{\hat{\sigma}}_{e}(t) \\
& =-\tilde{\boldsymbol{e}}^{\top}(t) Q \tilde{\boldsymbol{e}}(t)+2 \operatorname{tr}\left\{\tilde{\Lambda}^{\top}(t)\left[\boldsymbol{u}(t) \boldsymbol{\chi}^{\top}(t)-\gamma_{1}^{-1} \dot{\hat{\Lambda}}(t)\right]\right\}+2 \operatorname{tr}\left\{\tilde{K}_{x}^{\top}(t)\left[\boldsymbol{x}(t) \boldsymbol{\chi}^{\top}(t)-\gamma_{2}^{-1} \dot{\hat{K}}_{x}(t)\right]\right\} \\
& +2 \operatorname{tr}\left\{\tilde{K}_{r}^{\top}(t)\left[\boldsymbol{r}(t) \boldsymbol{\chi}^{\top}(t) \gamma_{3}^{-1} \dot{\hat{K}}_{r}(t)\right]\right\}+2 \operatorname{tr}\left\{\tilde{W}^{\top}(t)\left[\boldsymbol{\varphi}(t) \boldsymbol{\chi}^{\top}(t)-\gamma_{4}^{-1} \dot{\hat{W}}(t)\right]\right\} \\
& +2 \chi_{2}(t) \hat{\lambda}_{2}(t)\left[\tilde{\boldsymbol{q}}^{\top}(t) \boldsymbol{\xi}_{1}(t)+\tilde{\omega}(t) \xi_{21}(t)+\sigma_{e}(t)-\hat{\sigma}_{e}(t) \operatorname{sign}\left(\chi_{2}(t)\right)\right]+2 \boldsymbol{\chi}(t)^{\top}(\boldsymbol{\sigma}(t)-\boldsymbol{h}(t)) \\
& -2 \gamma_{5}^{-1}\left(\boldsymbol{\sigma}^{*}-\hat{\boldsymbol{\sigma}}(t)\right)^{\top} \dot{\hat{\boldsymbol{\sigma}}}(t)-2 \gamma_{6}^{-1} \tilde{\boldsymbol{q}}^{\top}(t) \dot{\hat{\boldsymbol{q}}}(t)-2 \gamma_{7}^{-1} \tilde{\omega}(t) \dot{\hat{\omega}}(t)-2 \gamma_{8}^{-1}\left(\sigma_{e}^{*}-\hat{\sigma}_{e}(t)\right) \dot{\hat{\sigma}}_{e}(t) \text {. }
\end{aligned}
$$

where we use the well known trace property $\operatorname{tr}\left(\boldsymbol{a}^{\top} \boldsymbol{b}\right)=\operatorname{tr}\left(\boldsymbol{b} \boldsymbol{a}^{\top}\right)$ for any vectors $\boldsymbol{a}$ and $\boldsymbol{b}$ of the same dimension. Substituting the adaptive laws and after some algebra we obtain

$$
\begin{aligned}
\dot{V}(t) & \leq-\tilde{\boldsymbol{e}}^{\top}(t) Q \tilde{\boldsymbol{e}}(t)+2 \boldsymbol{\chi}(t)^{\top}(\boldsymbol{\sigma}(t)-\boldsymbol{h}(t))-2\left(\boldsymbol{\sigma}^{*}-\hat{\boldsymbol{\sigma}}(t)\right)^{\top}|\boldsymbol{\chi}(t)|-2 \tilde{y}_{e}(t)\left[\tilde{\boldsymbol{q}}^{\top}(t) \boldsymbol{\xi}_{1}(t)+\tilde{\omega}(t) \xi_{21}(t)\right] \\
& +2 \chi_{2}(t) \hat{\lambda}_{2}(t)\left[\sigma_{e}(t)-\hat{\sigma}_{e}(t) \operatorname{sign}\left(\chi_{2}(t)\right)\right]-2\left(\sigma_{e}^{*}-\hat{\sigma}_{e}(t)\right)\left[\left|\tilde{y}_{e}(t)\right|+\hat{\lambda}_{2}(t)\left|\chi_{2}(t)\right|\right],
\end{aligned}
$$


where we have used the following properties of the projection operator ${ }^{17}$

$$
\begin{gathered}
\operatorname{tr}\left\{\tilde{\Lambda}^{\top}(t)\left[\boldsymbol{u}(t) \boldsymbol{\chi}^{\top}(t)-\Pi\left\{\hat{\Lambda}(t), \boldsymbol{u}(t) \boldsymbol{\chi}^{\top}(t)\right\}\right]\right\} \leq 0 \\
\tilde{\omega}(t)\left[\tilde{y}_{e}(t) \xi_{21}(t)+\hat{\lambda}_{2}(t) \chi_{2}(t) \xi_{21}(t)-\Pi\left\{\hat{\omega}(t), \tilde{y}_{e}(t) \xi_{21}(t)+\hat{\lambda}_{2}(t) \chi_{2}(t) \xi_{21}(t)\right\}\right] \leq 0,
\end{gathered}
$$

The terms $\boldsymbol{\chi}(t)^{\top}(\boldsymbol{\sigma}(t)-\boldsymbol{h}(t))-\left(\boldsymbol{\sigma}^{*}-\hat{\boldsymbol{\sigma}}(t)\right)^{\top}|\boldsymbol{\chi}(t)|$ are evaluated componentwise as follows

$$
\begin{aligned}
& \boldsymbol{\chi}(t)^{\top}(\boldsymbol{\sigma}(t)-\boldsymbol{h}(t))-\left(\boldsymbol{\sigma}^{*}-\hat{\boldsymbol{\sigma}}(t)\right)^{\top}|\boldsymbol{\chi}(t)|=\chi_{1}(t) \sigma_{1}(t)-\left|\chi_{1}(t)\right| \hat{\sigma}_{1}(t)-\left(\sigma_{1}^{*}-\hat{\sigma}_{1}(t)\right)\left|\chi_{1}(t)\right|+\chi_{2}(t) \sigma_{2}(t) \\
& -\left|\chi_{2}(t)\right| \hat{\sigma}_{2}(t)-\left(\sigma_{2}^{*}-\hat{\sigma}_{2}(t)\right)\left|\chi_{2}(t)\right|=\chi_{1}(t) \sigma_{1}(t)-\sigma_{1}^{*}\left|\chi_{1}(t)\right|+\chi_{2}(t) \sigma_{2}(t)-\sigma_{2}^{*}\left|\chi_{2}(t)\right| \chi_{2}(t) \leq 0 .
\end{aligned}
$$

Taking into account the positivity of the estimate $\hat{\lambda}_{2}(t)$ the last two terms are evaluated as follows

$$
\begin{aligned}
& \chi_{2}(t) \hat{\lambda}_{2}(t)\left[\sigma_{e}(t)-\hat{\sigma}_{e}(t) \operatorname{sign}\left(\chi_{2}(t)\right)\right]-\left(\sigma_{e}^{*}-\hat{\sigma}_{e}(t)\right)\left[\left|\tilde{y}_{e}(t)\right|+\hat{\lambda}_{2}(t)\left|\chi_{2}(t)\right|\right]=\sigma_{e}(t) \chi_{2}(t) \hat{\lambda}_{2}(t) \\
& -\sigma_{e}^{*}\left|\chi_{2}(t)\right| \hat{\lambda}_{2}(t)-\left(\sigma_{e}^{*}-\hat{\sigma}_{e}(t)\right)\left|\tilde{y}_{e}(t)\right| \leq-\left(\sigma_{e}^{*}-\hat{\sigma}_{e}(t)\right)\left|\tilde{y}_{e}(t)\right| \leq-\left(\sigma_{e}^{*}-\hat{\sigma}_{e}(t)\right) \tilde{y}_{e}(t) .
\end{aligned}
$$

Taking into account the inequalities above and the error definition (31) we obtain

$$
\dot{V}(t) \leq-\tilde{\boldsymbol{e}}^{\top}(t) Q \tilde{\boldsymbol{e}}(t)-\tilde{y}_{a}^{2}(t),
$$

which implies that the error signals $\tilde{\Lambda}(t), \tilde{K}_{x}(t), \tilde{K}_{r}(t), \tilde{W}(t), \boldsymbol{\sigma}^{*}-\hat{\boldsymbol{\sigma}}(t), \tilde{\boldsymbol{q}}(t), \tilde{\omega}(t)$ and $\sigma_{e}^{*}-\hat{\sigma}_{e}(t)$ are bounded. Then, the estimates $\hat{\Lambda}(t), \hat{K}_{x}(t), \hat{K}_{r}(t), \hat{W}(t), \hat{\boldsymbol{\sigma}}(t), \hat{\boldsymbol{q}}(t), \hat{\omega}(t)$ and $\hat{\sigma}_{e}(t)$ are bounded as well. At this moment we cannot conclude anything about the boundedness of $\hat{\boldsymbol{e}}(t), \boldsymbol{e}(t), \hat{y}_{e}(t), \tilde{y}_{e}(t)$ or $y_{e}(t)$. However, we can integrate the inequality (39) and obtain

$$
\int_{0}^{t}\left[\tilde{\boldsymbol{e}}^{\top}(\tau) Q \tilde{\boldsymbol{e}}(\tau)+\tilde{y}_{e}^{2}(\tau)\right] d \tau \leq V(0)-V(t) .
$$

From Lemma 1 it follows that $V(t)$ is bounded, therefore $\tilde{\boldsymbol{e}}(t) \in \mathcal{L}_{2}$ and $\tilde{y}_{e}(t) \in \mathcal{L}_{2}$.

We notice that Lemma 1 holds independent of specific control design, which we provide in the next subsection following the steps from Reference. ${ }^{19}$

\section{B. Control Signal}

Our goal is design aileron deflection and differential throttle commands $\delta_{a}(t)$ and $\Delta \delta_{T}(t)$ for the cascaded prediction system

$$
\begin{aligned}
\dot{\hat{\boldsymbol{e}}}(t) & =A_{m} \hat{\boldsymbol{e}}(t)+B\left[\hat{\Lambda}(t) \overline{\boldsymbol{u}}(t)+\hat{K}_{x}^{\top}(t) \boldsymbol{x}(t)+\hat{K}_{r}^{\top}(t) \boldsymbol{r}(t)+\hat{W}^{\top}(t) \boldsymbol{\varphi}(t)+\boldsymbol{h}(t)\right] \\
\varepsilon^{-1} \dot{\boldsymbol{\xi}}_{2}(t) & =\left(A_{0}-\boldsymbol{b}_{0} \boldsymbol{k}_{0}^{\top}\right) \boldsymbol{\xi}_{2}(t)+\boldsymbol{b}_{0} u_{e}(t),
\end{aligned}
$$

such that $\hat{\boldsymbol{e}}(t) \rightarrow 0$ as $t \rightarrow \infty$. We notice that the second system in (41) is of relative degree one and minimum phase, and the matrix $A_{0}-\boldsymbol{b}_{0} \boldsymbol{k}_{0}^{\top}$ is Hurwitz. Therefore the vector $\boldsymbol{k}_{0}$ can be chosen such that the transfer function from the input $u_{e}(t)$ to the output $\xi_{21}(t)$ has the form

$$
\xi_{21}(s)=\frac{\varepsilon \omega_{1}}{s+\varepsilon \omega_{2}} u_{e}(s)
$$

where $\omega_{1}>0$ and $\omega_{2} \neq 0$. The rest of the system is input to state stable, where the input is $\xi_{21}(t)$, and is bounded as long as $\xi_{21}(t)$ is bounded. Therefor the control design besides the tracking task must also guarantee the boundedness of $\xi_{21}(t)$. The cascaded system under the consideration takes the form

$$
\begin{aligned}
\dot{\hat{\boldsymbol{e}}}(t) & =A_{m} \hat{\boldsymbol{e}}(t)+B\left[\hat{\Lambda}(t) \overline{\boldsymbol{u}}(t)+\hat{K}_{x}^{\top}(t) \boldsymbol{x}(t)+\hat{K}_{r}^{\top}(t) \boldsymbol{r}(t)+\hat{W}^{\top}(t) \boldsymbol{\varphi}(t)+\boldsymbol{h}(t)\right] \\
\varepsilon^{-1} \dot{\xi}_{21}(t) & =-\omega_{1} \xi_{21}(t)+\omega_{2} u_{e}(t),
\end{aligned}
$$

Since $\hat{\Lambda}(t)$ is diagonal with positive entries, we can design the control signal $\overline{\boldsymbol{u}}(t)$ as follows

$$
\overline{\boldsymbol{u}}(t)=\hat{\Lambda}^{-1}(t)\left[-\hat{K}_{x}^{\top}(t) \boldsymbol{x}(t)-\hat{K}_{r}^{\top}(t) \boldsymbol{r}(t)-\hat{W}^{\top}(t) \boldsymbol{\varphi}(t)-\boldsymbol{h}(t)\right] .
$$


Whereas the signal

$$
\delta_{a}(t)=\bar{u}_{1}(t)+\delta_{a}^{0}(t),
$$

where $\delta_{a}^{0}(t)$ is the baseline signal from (16) is the actual aileron deflection command, the signal $\Delta T(t)=$ $\bar{u}_{2}(t)+\Delta T^{0}(t)$, where $\Delta T^{0}(t)$ is the baseline signal defined in (16), represents differential thrust command to the engine dynamics. Since we will design the differential throttle command from the point of view of controlling the model (52), the stabilizing function is designed as follows

$$
\mu(t)=\frac{\bar{u}_{2}(t)-\hat{\boldsymbol{q}}^{\top}(t) \boldsymbol{\chi}_{1}(t)-\hat{\sigma}_{e}(t) \operatorname{sign}\left(\chi_{2}(t)\right)}{\hat{\omega}(t)},
$$

which is well defined since $\hat{\omega}(t)>0$ is guaranteed by the adaptive law (32). Next we filter the stabilizing function $\mu_{1}(t)$ through a second order stable filter

$$
\begin{aligned}
\varepsilon^{-1} \dot{z}_{1}(t) & =\omega_{f} z_{2}(t) \\
\dot{z}_{2}(t) & =-2 \zeta_{f} \omega_{f} z_{2}(t)-\omega_{f}\left[z_{1}(t)-\mu(t)\right],
\end{aligned}
$$

and introduce an error variable $\eta(t)=\xi_{21}(t)-z_{1}(t)$. The tracking error prediction model now can be written as

$$
\dot{\hat{\boldsymbol{e}}}(t)=A_{m} \hat{\boldsymbol{e}}(t)+\boldsymbol{b}_{2} \hat{\lambda}_{2}(t) \hat{\omega}(t) \eta(t)+\boldsymbol{b}_{2} \hat{\lambda}_{2}(t) \hat{\omega}(t)\left[z_{1}(t)-\mu(t)\right],
$$

where $\boldsymbol{b}_{2}$ is the second column of matrix $B$. Filtering the auxiliary error term $\hat{\lambda}_{2}(t) \hat{\omega}(t)\left[z_{1}(t)-\mu(t)\right]$ through the stable filter

$$
\dot{\boldsymbol{\zeta}}(t)=A_{m} \boldsymbol{\zeta}(t)+\boldsymbol{b}_{2} \hat{\omega}(t) \hat{\eta}_{1}(t)\left[z_{1}(t)-\mu(t)\right],
$$

we introduce the compensated error $\overline{\boldsymbol{e}}(t)=\hat{\boldsymbol{e}}(t)-\boldsymbol{\zeta}(t)$ which satisfies the dynamic equation

$$
\dot{\overline{\boldsymbol{e}}}(t)=A_{m} \overline{\boldsymbol{e}}(t)+\boldsymbol{b}_{2} \hat{\lambda}_{2}(t) \hat{\omega}(t) \eta(t) .
$$

Differentiating $\eta(t)$ we obtain

$$
\varepsilon^{-1} \dot{\eta}(t)=-\varepsilon^{-1} \omega_{1} \xi_{21}(t)+\omega_{2} u_{e}(t)-\omega_{f} z_{2}(t),
$$

which together with the compensated error dynamics (49) make a singular perturbation problem ${ }^{13}$

$$
\begin{aligned}
\dot{\bar{e}}(t) & =A_{m} \overline{\boldsymbol{e}}(t)+\boldsymbol{b}_{2} \hat{\lambda}_{2}(t) \hat{\omega}(t) \eta(t) \\
\varepsilon^{-1} \dot{\eta}(t) & =-\omega_{1} \xi_{21}(t)+\omega_{2} u_{e}(t)-\omega_{f} z_{2}(t) .
\end{aligned}
$$

In Reference ${ }^{19}$ we prove that the singular two time scale separation principle can be applied to the cascaded system $(52)$ and the control signal $u_{e}(t)$ can be designed through from the perspective of controlling the reduced system

$$
\frac{d \eta(\tau)}{d \tau}=-\omega_{f} z_{2}(t)-\omega_{1} \xi_{21}(t)+\omega_{2} u(t) .
$$

in the time scale $\tau=\varepsilon t$. The boundary layer system in this case has the form

$$
\dot{\boldsymbol{y}}(t)=A_{m} \boldsymbol{y}(t),
$$

where $\boldsymbol{y}(t)=\boldsymbol{e}(t)-A_{m}^{-1} \boldsymbol{b}_{2} \hat{\lambda}_{2}(t) \hat{\omega}(t) \eta(t)$, and is exponentially stable. The control signal is designed as

$$
u_{e}(t)=\frac{-c \mu(t)+\left(\omega_{1}-c\right) \xi_{21}(t)+\omega_{f} z_{2}(t)}{\omega_{2}},
$$

and exponentially stabilizes reduced system (53). 
Then it can be proved following the Reference ${ }^{19}$ that the solutions of the cascaded system (52 and the following system

$$
\begin{aligned}
\dot{\hat{\boldsymbol{e}}}(t) & =A_{m} \hat{\boldsymbol{e}}(t)+\boldsymbol{b}_{2} \hat{\lambda}_{2}(t) \hat{\omega}(t) \eta(t)+\boldsymbol{b}_{2} \hat{\lambda}_{2}(t) \hat{\omega}(t)\left[z_{1}(t)-\mu(t)\right] \\
\varepsilon^{-1} \dot{\eta}(t) & =-\omega_{1} \xi_{21}(t)+\omega_{2} u_{e}(t)-\omega_{f} z_{2}(t) .
\end{aligned}
$$

satisfies the relationship

$$
\eta(t)=O(\varepsilon)
$$

for all $t>0$, and the relationship

$$
\begin{aligned}
& \hat{\boldsymbol{e}}(t)=O(\varepsilon)+O\left(\omega_{f}^{-1}\right) \\
& \overline{\boldsymbol{e}}(t)=O(\varepsilon)
\end{aligned}
$$

for all $t>t^{*}$, where $t^{*}$ depends on the convergence rate of the compensated error system (52) in faster time scale, and on $\varepsilon$. From the boundedness of $\hat{\boldsymbol{e}}(t)$ and $\eta(t)$ and from the inequality (40) it follows thate $(t)$, $\hat{y}_{e}(t)$ and $y_{e}(t)$ are bounded. Then, from the dynamics (27) it follows that $\dot{\tilde{\boldsymbol{e}}}(t)$ is bounded. Therefore, the application of Barbalat's lemma results in $\tilde{\boldsymbol{e}}(t) \rightarrow 0$ as $t \rightarrow \infty$. From this convergence one can infer that the relationship

$$
\boldsymbol{e}(t)=O(\varepsilon)+O\left(\omega_{f}^{-1}\right)
$$

holds as $t \rightarrow \infty$.

Since the lateral-directional tracking error $\mathbf{D}(\mathbf{t})$ is bounded, from the boundedness of the reference command $\boldsymbol{r}_{D}(t)$ it follows the the lateral-directional variables are bounded, and the bounds are independent of the unknown parameters. That is the compact set $\Omega_{\boldsymbol{x}_{D}}$ can be chosen independent of the adaptive scheme such that $\boldsymbol{x}_{D}(T) \in \Omega_{\boldsymbol{x}_{D}}$ for all $t \geq 0$. From this considerations and Remark 3 follows the validity of the approximation (21). This completes the proof of the following theorem.

Theorem 1 . Let the outer loop variables $\boldsymbol{x}_{L}$ be in the compact set $\Omega_{\boldsymbol{x}_{L}}$. Then the differential throttle command $\Delta \delta_{T}(t)$ given by the equation (55), along with the aileron deflection command $\delta_{a}(t)$ (45), adaptive laws (26), (32), stabilizing function (46) and filters (47), (49), (50) regulates the error signal $\boldsymbol{e}_{D}(t)$ to a neighborhood of the origin with the diameter proportional to $\varepsilon$ and inversely proportional to the filters' frequency $\omega_{f}$.

\section{Simulation Results}

For the simulation we consider a damaged GTM model that before the damage was in straight and level flight at $h=30000 \mathrm{ft}$ with speed $M=0.8$. We consider only linearized lateral-directional dynamics of the vertical tail loss GTM model, since the longitudinal dynamics do not change much and the model ia still symmetric about the vertical plane. The linearized lateral directional system is given by the matrices

$$
A_{D}=\left[\begin{array}{cccc}
-0.0794 & -0.9975 & 0.0496 & 0.0404 \\
-0.5646 & -0.0955 & -0.0217 & 0 \\
-7.4175 & 0.2784 & -1.7186 & 0 \\
0 & 0.0507 & 1.0000 & 0
\end{array}\right], B_{D}=\left[\begin{array}{cc}
0.0024 & 0.0000 \\
-0.0523 & 0.0293 \\
6.4318 & 0.0010 \\
0 & 0
\end{array}\right] .
$$

It is unstable with the poles at $(-1.6473,-0.7007,0.3683,0.0862)$. The engines are given by the linear system

$$
\varepsilon=0.25, A_{e}=\left[\begin{array}{cccc}
0 & 1 & 0 & 0 \\
0 & 0 & 1 & 0 \\
0 & 0 & 0 & 1 \\
-150.0135 & -171.4720 & -73.4960 & -14.0000
\end{array}\right], \boldsymbol{b}_{e}=\left[\begin{array}{c}
0 \\
0 \\
0 \\
150.0135
\end{array}\right],
$$

which correspond to the normalized thrust and normalized throttle input. The engine dynamic model has a settling time of $t_{s}=10.3851 \mathrm{sec}$. 
We run a simulation assuming that the systems are known, but engine states are not accessible and only thrust output $T_{n}(t)$ is available for feedback. The engine dynamics are written in the equivalent form

$$
\begin{aligned}
\dot{z}_{1}(t) & =z_{2}(t) \\
\dot{z}_{2}(t) & =z_{3}(t) \\
\dot{z}_{3}(t) & =z_{4}(t) \\
\dot{z}_{4}(t) & =-0.5860 z_{1}(t)-2.6793 z_{2}(t)-4.5935 z_{3}(t)-3.5 z_{4}(t)+0.5860 u_{n}(t) \\
T_{n}(t) & =z_{1}(t)
\end{aligned}
$$

for which an observer is constructed with the poles at $(-8.9,-8.8,-8.7,-8.6)$, and the states of the observer are used in generating the differential thrust as well as the aileron deflection commands.

In the first simulation we stabilize the airplane given from initial conditions $\beta(0)=1.5^{\circ}, r(0)=0, p(o)=$ $0, \phi(0)=0$. The simulation results are presented in Figures 3(a), 3(b), 4(a) and 4(b).
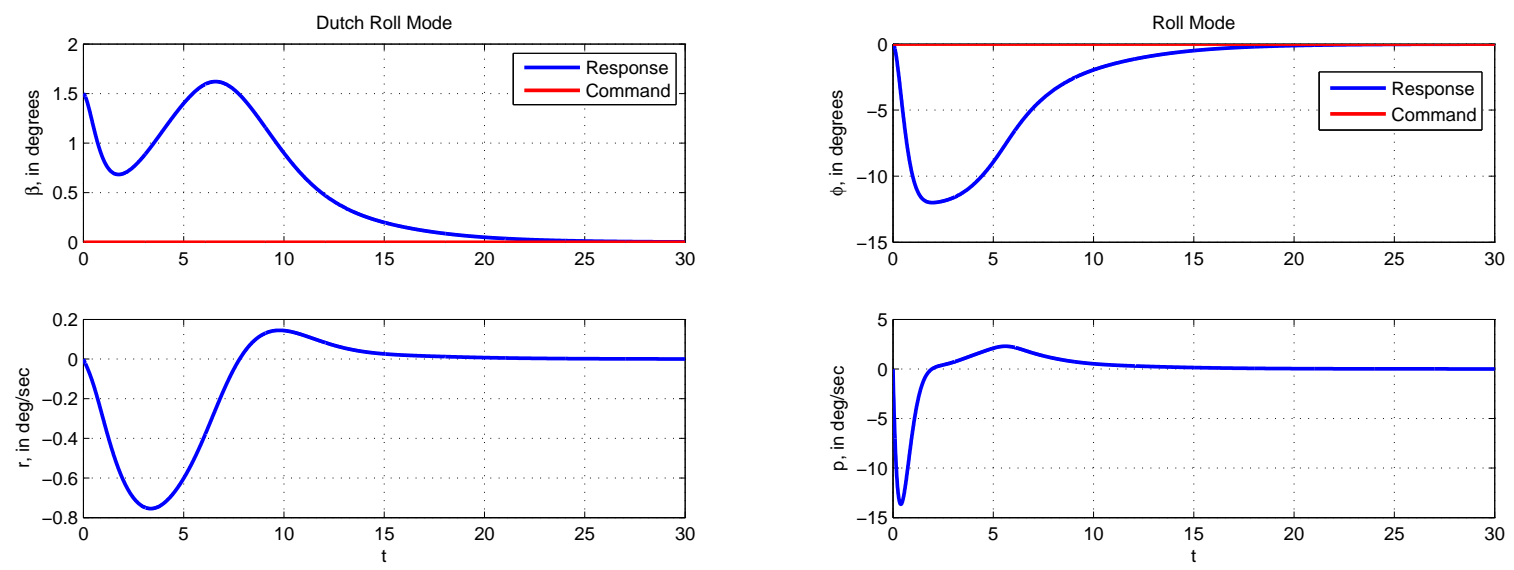

(a) Sideslip angle and yaw rate

(b) Roll angle and roll rate

Figure 3. Lateral directional performance of the airplane
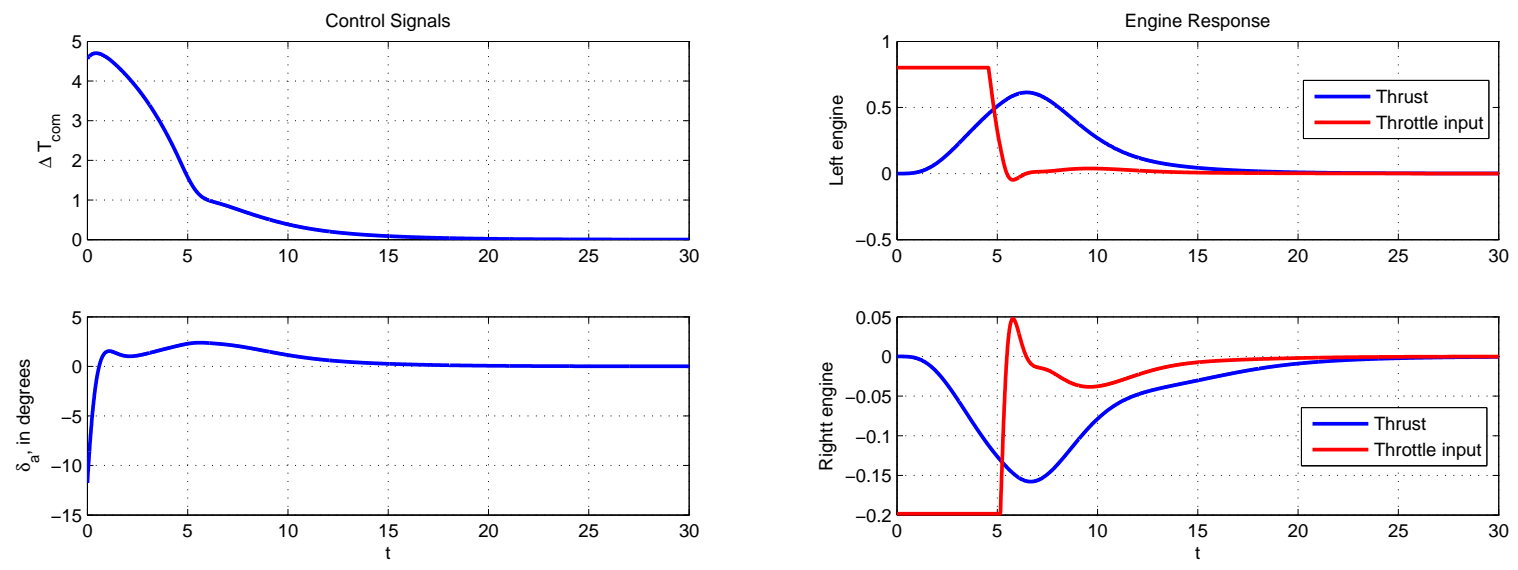

(a) Differential Thrust and Aileron deflection commands

(b) Engine model performance

Figure 4. Stabilizing control signals

It is evident from the figures that the stabilization is achieved with the chosen engine model. The initial rapid reduction in sideslip angle is due to aileron deflection at the initial period as can be seen from Figure 4(a). This also results in the negative banking of the airplane (Figure 3(b)), which buys some time for the engines to generate a differential thrust. However, the thrust generated it is still not sufficient enough to 
decrease the sideslip angle. When the aileron returns to a small region around equilibrium, the sideslip angle increases again for the next $10 \mathrm{sec}$, but the engines are able to generate enough torque to stop the increase and eventually regulate the sideslip angle.

The differential thrust command is large during the initial period and results in the saturation of throttle inputs for both engines as can be seen in Figure 4(b). The engine responses are slow, but sufficient enough to compensate for the chosen initial disturbance. It should be mentioned that the airplane departs from the course during the recovery. After a stabile flight regime is established, the course can be changed, which we demonstrate in the next simulation.

The next simulation results show the tracking performance of the airplane with initial $-0.5^{\circ}$ sideslip angle following the $15^{\circ}$ bank angle command while regulating the sideslip. The performance is displayed in Figures 5(a), 5(b), 6(a) and 6(b).
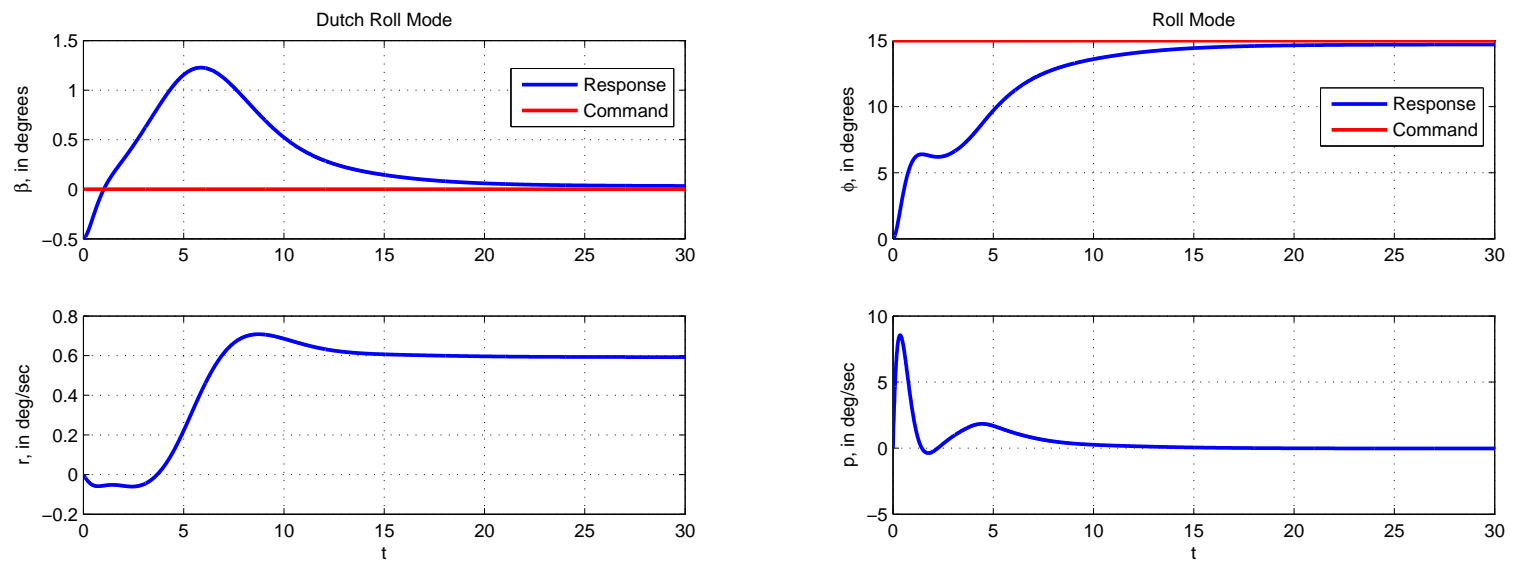

(a) Sideslip angle and yaw rate

(b) Roll angle and roll rate

Figure 5. Tracking performance of the airplane
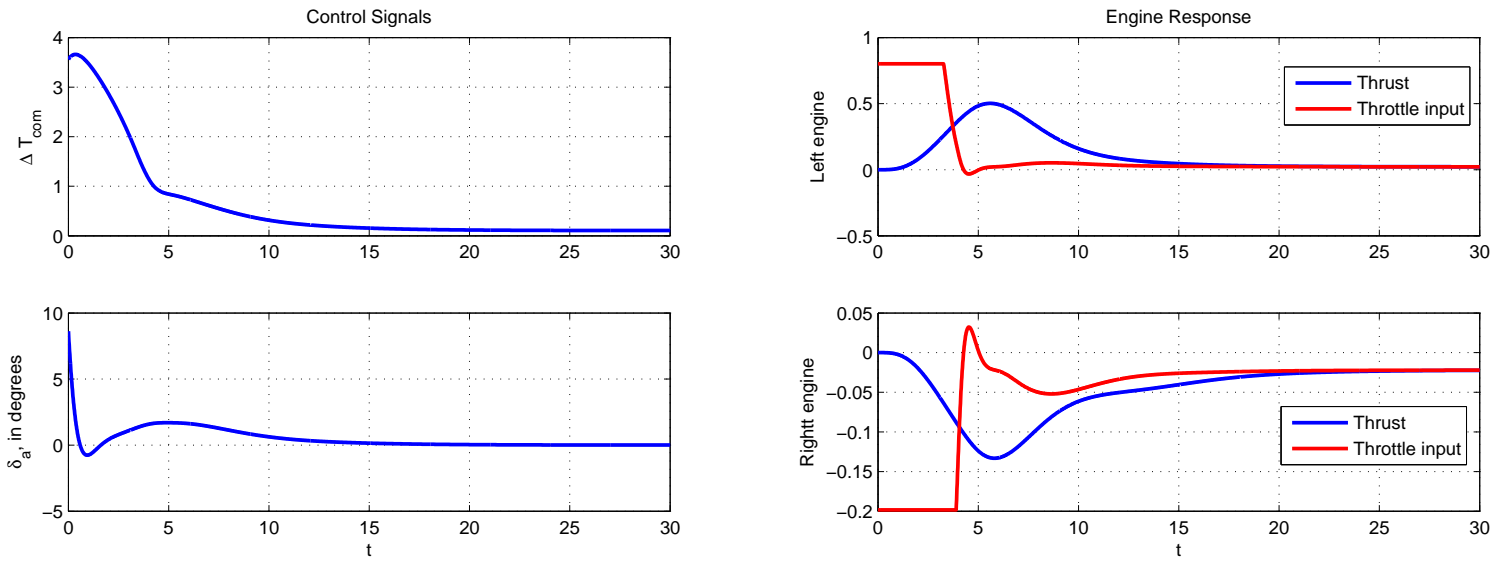

(a) Differential Thrust and Aileron deflection commands

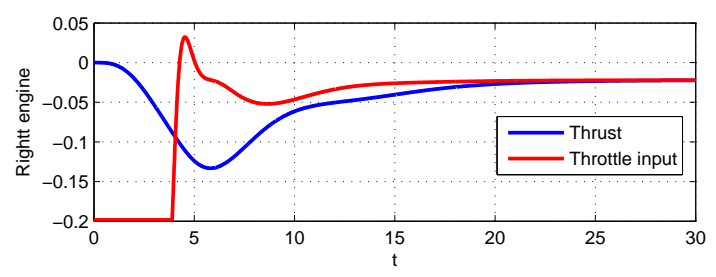

(b) Engine model performance

Figure 6. Tracking control signals

It can be seen from the figures that the proposed control scheme has also tracking capabilities. The steady turn rate is about $0.6^{\circ}$ per second, which is sufficient enough to change the course of the given flight conditions as required. The required differential thrust is again higher in the initial period and results in the throttle saturation of both engines 4(b).

We run the last simulation to stabilize the airplane with the proposed adaptive control when the engine dynamics are assumed to be unknown, but the relative degree is known. The results for the initial conditions $\beta(0)=1.5^{\circ}, r(0)=0, p(o)=0, \phi(0)=0$ are displayed in Figures 5(a), 5(b), 6(a) and 6(b). Although the 

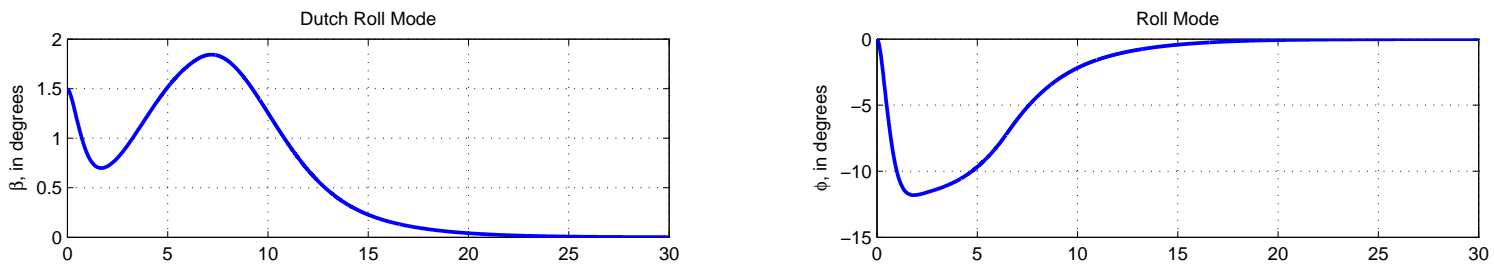

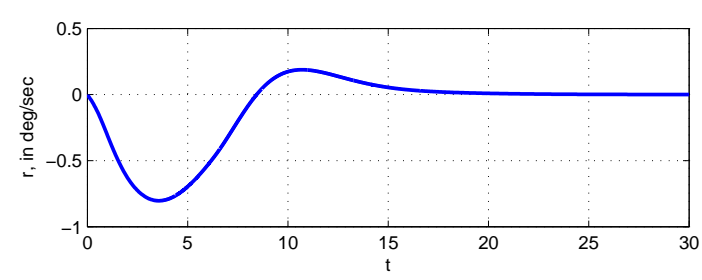

(a) Sideslip angle and yaw rate

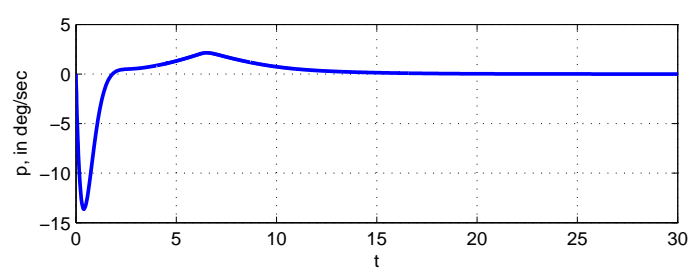

(b) Roll angle and roll rate

Figure 7. Adaptive stabilization of the vertical tail loss airplane
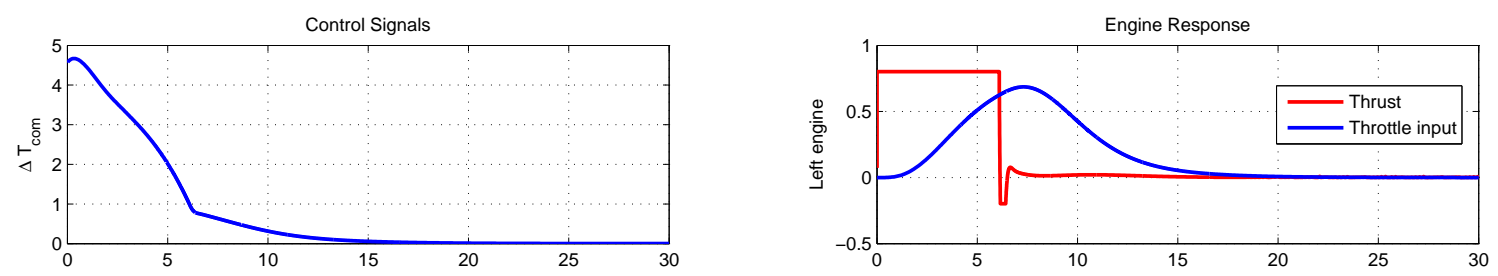

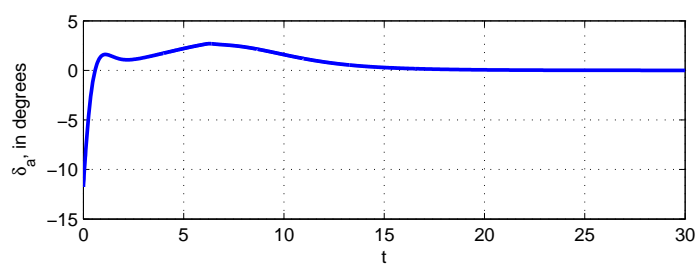

(a) Differential Thrust and Aileron deflection commands

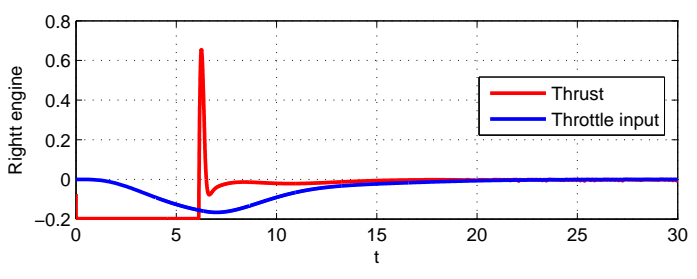

(b) Engine model performance

Figure 8. Adaptive control signals

convergence is somewhat slower than in the known case, the overall performance is similar and the airplane behaves in a similar way.

\section{Conclusion}

We considered a control problem for the vertical tail loss generic transport aircraft model, using the engines' differential thrust in place of a rudder, which is assumed to be lost. Although the required differential trust generation is substantially slower than the conventional yaw moment generation by the rudder deflection, we show that the aircraft can still be locally controlled, using the proposed control algorithm. This approach is demonstrated in the simulations for both stabilization of the damaged airplane and tracking of a given command. Future validation of the approach will be conducted using full nonlinear aircraft simulations with realistic and accurate engine model.

\section{Acknowledgments}

The authors wish to thank Mark Sabugal of NASA Ames Research Center for his contribution in engine model development. 


\section{References}

${ }^{1}$ No Flight Controls. Aviation Week and Space Technology, 159(23):42-43, 2003.

${ }^{2}$ National Transportation Safety Board. In-Flight Separation of Vertical Stabilizer American Airlines Flight 587, Airbus Industries A300-605R, N14053 Belle Harbor, New York, November 12, 2001. NTSB/AAR-04/04, 2004.

${ }^{3}$ G. Bramesfeld, M. Maughmer, and S. Willits. Piloting Strategies for Controlling A Transport Aircraft After vertical Tail Loss. Journal of Aircraft, 43(1):216-225, 2006.

${ }^{4}$ F. W. Burcham, T. A. Maine, and J. J. Burken. Using Engine Thrust for Emergency Flight Control: MD-11 and B-747 Results. NASA/TM-1998-206552, May 1998.

${ }^{5}$ C. Cao and N. Hovakimyan. Design and Analysis of a Novel $\mathcal{L}_{1}$ Adaptive Control Architecture with Guaranteed Transient Performance. IEEE Trans. Autom. Contr., 53(2):586-591, 2008.

${ }^{6}$ C. Cao, N. Hovakimyan, I. Kaminer, V. V. Patel, and V. Dobrokhodov. Stabilization of Cascaded Systems Via $\mathcal{L}_{1}$ Adaptive Controller With Application to a UAV Path Folloing and Flight Test. In Proc. of the American Control Conference, pages $1787-1792,2007$.

${ }^{7}$ L. Crider. Control of Comertial Aircraft with Vertical Tail Loss. In Proceedings of AIAA 4th Aviation Technology, Integration and Operation Forum, AIAA 2004-6293., 2004.

${ }^{8}$ J. Csank, T.-H. Guo, and J. Litt. Simplified Engine Model. Integrated Resilient Aircraft Control Project, NASA Internal Software Release, 2009.

${ }^{9}$ G. B. Gilyard, J. L. Conley, J. Le, and Jr. F. W. Burcham. A Simulation Evaluation of a Four-Engine Jet Transport Using Engine Thrust Modulation for Flightpath Control. In Proc. of the AIAA Guidance, Navigation, and Control Conference,AIAA 1991-2233, 1991.

${ }^{10} \mathrm{M}$. Harefors and D. G. Bates. Integrated Propilsion-based Flight Control System Design for Civil Transport Aircraft. In Proceedings of IEEE Conference on Conrol Applications, Glasgow, Scotland, UK, pages 132-137, 2002.

${ }^{11}$ S. Haykin. Neural Networks: A Comprehensive Foundation. Prentice Hall, NJ, 1999.

${ }^{12}$ N. Hovakimyan, E. Lavretsky, and C. Cao. Adaptive Dynamic Inversion via Time-Scale Seperation. IEEE Trans. Neural Networks, 19(10):1702-1711, 2008.

${ }^{13}$ H.K. Khalil. Nonlinear Systems, Third Edition. Prentice Hall, New Jersey, 2002.

${ }^{14}$ Y. Liu, X. Tang, G. Tao, and S. M. Joshi. Adaptive Failure Compensation for Aircraft Tracking Control Using Engine Differential Based Mode. In Proc. of the American Control Conference,Minneapolis, MN, pages 5984-5989, 2006.

${ }^{15}$ K.S. Narendra and A.M. Annaswamy. Stable Adaptive Control. Prentice Hall, 1989.

${ }^{16}$ N. Nguyen, K. Krishnakumar, J. Kaneshige, and P. Nespeca. Flight Dynamics and Hybrid Adaptive Control of Damaged Aircraft. AIAA Journal of Guidance, Control, and Dynamics, 31(3):751-764, 2008.

${ }^{17}$ J. B. Pomet and L. Praly. Adaptive Nonlinear Regulation: Estimation from the Lyapunov Equation. IEEE Trans. Autom. Contr., 37(6):729-740, 1992.

${ }^{18}$ J.J. Slotine and W. Li. Applied Nonlinear Control. Prentice Hall, New Jersey, 1991.

${ }^{19} \mathrm{~V}$. Stepanyan. Control of Systems With Slow Actuators Using Time Scale Separation. In Proc. of the AIAA Guidance, Navigation, and Control Conference, 2009.

${ }^{20}$ V. Stepanyan, K. Krishnakumar, and N. Nguyen. Transient Performance and Asymptotic Tracking with Filtering Robust Adaptive Control. In Proc. of the In Proc. of the IEEE Aerospace Conference, 2009.

${ }^{21}$ N. Tanaka, S. Suzuki, K. Masui, and H. Tomita. Restructurable Guidance and Control for Aircraft with Failures Considering Gust Effect. AIAA Journal of Guidance, Control, and Dynamics, 29(3):671-679, 2006.

${ }^{22}$ K. A. Wise, E. Lavretsky, N. Hovakimyan, C. Cao, and J. Wang. Verifiable Adaptive Flight Control: UCAV and Aerial Refueling. In Proc. of the AIAA Guidance, Navigation, and Control Conference,AIAA 2008-6658, 2008. 